\title{
Standing Waves in a Rectangular Resonator Containing Acoustically Active Gases
}

\author{
Anna PERELOMOVA \\ Faculty of Applied Physics and Mathematics \\ Gdansk University of Technology \\ Narutowicza 11/12, 80-952 Gdańsk, Poland; e-mail: anpe@mif.pg.gda.pl
}

(received May 20, 2015; accepted October 19, 2015)

\begin{abstract}
The distribution of perturbations of pressure and velocity in a rectangular resonator is considered. A resonator contains a gas where thermodynamic processes take place, such as exothermic chemical reaction or excitation of vibrational degrees of a molecule's freedom. These processes make the gas acoustically active under some conditions. We conclude that the incident and reflected compounds of a sound beam do not interact in the leading order in the case of the periodic sound with zero mean pressure including waveforms with discontinuities. The acoustic field before and after forming of discontinuities is described. The acoustic heating or cooling in a resonator is discussed.
\end{abstract}

Keywords: standing waves; acoustically active gas; resonator; nonlinear propagation of sound.

PACS no 43.25, 43.35.Fj.

\section{Introduction}

The boundaries of a vessel over which sound spreads have a significant impact on the distribution of acoustic perturbations in the volume of the vessel. In contrast to a free space, the presence of boundaries determines the discrete spectrum of wave numbers of perturbations and may result in the standing waves in the volume of the resonator. The physically valid conditions on the boundaries of the resonator determine the magnitude and phase of the reflected waves. In general, intersecting nonlinear waves do interact. Many studies are devoted to wave fields in resonators of different shapes, first of all, those filled with the Newtonian fluids (Chester, 1964; Mortell et al., 2009; Biwa, Yazaki, 2010; Keller, 1977). Kaner, RudENKo, KHOKHLOV (1977) introduced an analytical method which applies different scales in description of the temporal evolution of standing waves in one-dimensional resonators. This method was successfully applied in studies of quasi-planar, weakly nonlinear, and weakly damping sound in non-dispersive flows (Rudenko, Soluyan, 2005). It imposes a slow dependence of the shape of progressive waves and their fast dependence on the retarded time. In connection to this waveforms before and after forming of discontinuities may be considered individually. In this way, it becomes possible to simplify wave equations in many cases. As for the perturbations in a one-dimensional resonator, the method makes it possible to subdivide the wave field into a sum of non-interacting planar waves which travel in opposite directions and to consider them individually. The total field represents a superposition of these planar waves. This is conditioned by their periodicity and zero mean pressure (OCHMANN, 1985). Shock waves in the resonators filled with Newtonian fluids have been considered in BIWA, YAZAKI (2010) and KeLLER (1977).

In the last decades, the interest to the nonNewtonian fluids and media with irreversible thermodynamic processes constantly grows ( $\mathrm{CHU}, 1970$; Parker, 1972; Makaryan, Molevich, 2007). The non-equilibrium molecular physics began to develop quickly due to the laser revolution in physics and chemistry (Osipov, Uvarov, 1992; Clarke, McChesNEY, 1976). Since that, the attention is concentrated on media which may reveal anomalous dispersion and attenuation (BAUER, BASs, 1973; Molevich, 2001). Under some conditions, the bulk viscosity of a fluid takes negative values and sound increases its magnitude in the course of propagation. As far as the author knows, a weakly nonlinear propagation of sound in a resonator which is filled with a relaxing gas, where relaxation may be irreversible, is a new subject of stud- 
ies. As well as in the case of resonators containing a Newtonian fluid, the planar waves, both incident and reflected, do not interact in the leading order in the volume of a resonator, if they are periodic and zero on average (Sec. 3). The standing acoustic waves in a rectangular resonator are considered before and after formation of discontinuities for two examples of thermodynamic relaxation in gases. The generic parameter which describes thermodynamic processes in a gas, $B$, is positive in an acoustically active gas and negative otherwise. The Newtonian attenuation, that is, mechanical viscosity of a gas and its thermal conduction, will be ignored. Shock waves with discontinuities in the wave profile always appear in a planar wave which propagates in an unbounded volume of an acoustically active medium, as well as in a resonator. The sound's magnitude increases in the course of sound propagation in the unbounded medium, but the nonlinear attenuation on the front of the saw-tooth wave leads to stabilization of the peak magnitude of the shock wave which tends to some positive value depending on $B$ as the time increases (PERELOMOVA, 2012). The total field in a resonator represents a sum of incident and reflected waves, which at first stage do not contain discontinuities. However, they necessarily appear as, the time increases; the time of formation is conditioned by $B$, the Mach number and parameter of nonlinearity of a gas. In the equilibrium regime with normal dispersion and attenuation, the magnitude of acoustic field in a resonator gets smaller, and discontinuity may not form at all (PERElomova, 2012). The non-linear generation of non-acoustic modes, such as the entropy and vorticity modes, is anomalous in the acoustically active gases. In a resonator, these effects accumulate over time. In particular, cooling of a medium takes place instead of heating (Sec. 4).

\section{Examples: gases with excited vibrational degrees of molecule's freedom and gases with chemical reactions}

The first example relates to a gas whose steady state is maintained by pumping energy into the vibrational degrees of molecule's freedom (OsIPov, Uvarov, 1992; Clarke, McChesney, 1976). The quantity

$$
B=-\frac{(\gamma-1)^{2} T_{0}}{2 c^{3}}\left(\frac{C_{v}}{\tau_{R}}+\frac{\varepsilon-\varepsilon_{e q}}{\tau_{R}^{2}} \frac{\mathrm{d} \tau}{\mathrm{d} T}\right)
$$

is positive in the acoustically active gas and negative otherwise. It is evaluated at unperturbed pressure and temperature of a gas, $p_{0}, T_{0}$, where $\gamma$ is the specific heats ratio for an ideal gas, $c$ is the equilibrium speed of the sound of an infinitely small magnitude, $C_{v}=\mathrm{d} \varepsilon_{e q} / \mathrm{d} T$ is the equilibrium specific heat at a constant volume $\left(\varepsilon_{e q}\right.$ is the equilibrium value of the vibrational energy, $\varepsilon$ ), and $\tau_{R}$ denotes the vibrational relaxation time. The relaxation time in the most important cases may be thought of as a function exclusively of the temperature (OSIPOV, UVAROV, 1992; ZELDOVICH, RAIZER, 1966). The non-equilibrium excitation is possible in principle due to the negative $\mathrm{d} \tau_{R} / \mathrm{d} T . B$ in Eq. (1) is the decrement (or increment, if positive) of acoustic magnitudes in the highfrequency oscillations, when $\omega \tau_{R} \gg 1$, where $\omega$ denotes the characteristic frequency of the sound. In the low-frequency regime, the attenuation (or enhancement) of the sound is insignificant (PERELOMOVA, 2010).

In the case of gases in which exothermic chemical reaction occurs (BAUER, BAss, 1973; MOLEviCH, 2001),

$$
B=\frac{Q_{0}(\gamma-1)\left(Q_{\rho}+(\gamma-1) Q_{T}\right)}{2 c^{2} m}
$$

is the quantity evaluated at unperturbed $p_{0}, T_{0}, Y_{0}$, where $Y$ denotes the mass fraction of a reagent $A^{*}$ in $A^{*} \rightarrow B^{*}$ exothermic reactions; $Q$ is the heat produced in a medium per one molecule due to a chemical reaction, $Q_{0}=Q\left(T_{0}, \rho_{0}, Y_{0}\right)$, and $m$ is the molecule's mass. The dimensionless quantities $Q_{T}, Q_{\rho}$ are determined by means of partial derivatives of the heat produced due to a chemical reaction with respect to the temperature and density of the mixture, respectively:

$$
\begin{aligned}
Q_{T} & =\frac{T_{0}}{Q_{0}}\left(\frac{\partial Q}{\partial T}\right)_{T_{0}, \rho_{0}, Y_{0}}, \\
Q_{\rho} & =\frac{\rho_{0}}{Q_{0}}\left(\frac{\partial Q}{\partial \rho}\right)_{T_{0}, \rho_{0}, Y_{0}} .
\end{aligned}
$$

The characteristic time of chemical reaction is

$$
\tau_{c}=\frac{H m Y_{0}}{Q_{0} Q_{Y}}
$$

where $H$ is the reaction enthalpy per unit mass of reagent $A^{*}, Q_{Y}=\frac{Y_{0}}{Q_{0}}\left(\frac{\partial Q}{\partial Y}\right)_{T_{0}, \rho_{0}, Y_{0}} . B$ given by Eq. (2) is the decrement (or increment, if positive) of acoustic magnitudes if $\omega \tau_{c} \gg 1$. In the low-frequency regime, as well as in the case of vibrationally excited gases, the attenuation (or enhancement) of the sound is insignificant. At low frequencies, a gas has sufficient time to relax over the sound period and behaves like a Newtonian fluid. This applies to both considered cases of relaxation.

\section{Wave perturbations in a waveguide}

An equation which describes dynamics of the potential of velocity $(\varphi: \nabla \varphi=\mathbf{v})$ in gases with type 
of relaxation described in the previous section, in the leading order is

$$
\begin{aligned}
\frac{\partial^{2} \varphi}{\partial t^{2}}-c^{2} \Delta \varphi-2 c B \frac{\partial \varphi}{\partial t}= & -2 \nabla \varphi\left(\nabla \frac{\partial \varphi}{\partial t}\right) \\
& -(\gamma-1) \Delta \varphi \frac{\partial \varphi}{\partial t} .
\end{aligned}
$$

This wave equation includes the term responsible for dispersion (it is proportional to $B$ ) and differs from the well-known equation describing lossless flows. Even in the case of a lossless perfect gas, no analytical solutions are available for the unsteady flow apart from those for planar waves (HAMilton, MoRfEy, 1998). We will consider the velocity potential in a resonator consisting of four parts specifying periodic planar waves:

$$
\begin{array}{ll}
\varphi_{1}\left(\tau_{1}, \vartheta=\mu t\right), & \varphi_{2}\left(\tau_{2}, \vartheta\right), \\
\varphi_{3}\left(\tau_{3}, \vartheta\right), & \varphi_{4}\left(\tau_{4}, \vartheta\right),
\end{array}
$$

where $\mu$ is a generic small parameter that characterizes slow variations in the waveforms due to nonlinearity and relaxation, $k_{x}, k_{y}$ are components of the wave vector, and $\omega=c \sqrt{k_{x}^{2}+k_{y}^{2}}$, and

$$
\begin{array}{ll}
\tau_{1}=t-\frac{k_{x}}{\omega} x+\frac{k_{y}}{\omega} y, & \tau_{2}=t-\frac{k_{x}}{\omega} x-\frac{k_{y}}{\omega} y, \\
\tau_{3}=t+\frac{k_{x}}{\omega} x+\frac{k_{y}}{\omega} y, & \tau_{4}=t+\frac{k_{x}}{\omega} x-\frac{k_{y}}{\omega} y .
\end{array}
$$

Our primary objective is to derive model equations valid at order $\mu^{2}$ for sound perturbations in a resonator. Equation (5) with account for (6) may be rewritten in the leading order as follows:

$$
\begin{gathered}
2 \mu \sum_{i=1}^{4} \frac{\partial^{2} \varphi_{i}}{\partial \tau_{i} \partial \vartheta}-2 c B \sum_{i=1}^{4} \frac{\partial \varphi_{i}}{\partial \tau_{i}}=-\frac{\gamma-1}{c^{2}}\left(\sum_{i=1}^{4} \frac{\partial^{2} \varphi_{i}}{\partial \tau_{i}^{2}}\right) \\
\cdot\left(\sum_{i=1}^{4} \frac{\partial \varphi_{i}}{\partial \tau_{i}}\right)-2\left(\sum_{i=1}^{4} \frac{\partial \varphi_{i}}{\partial \tau_{i}} \frac{\partial^{2} \varphi_{i}}{\partial \tau_{i}^{2}}\right. \\
\left.+\sum_{i, j=1, i \neq j}^{4} \frac{\alpha_{i, j} k_{x}^{2}+\beta_{i, j} k_{y}^{2}}{\omega^{2}} \frac{\partial \varphi_{i}}{\partial \tau_{i}} \frac{\partial^{2} \varphi_{j}}{\partial \tau_{j}^{2}}\right),
\end{gathered}
$$

where $\alpha_{1,2}=-\beta_{1,2}=1, \alpha_{1,3}=-\beta_{1,3}=-1, \alpha_{1,4}=$ $\beta_{1,4}=-1, \alpha_{2,3}=\beta_{2,3}=-1, \alpha_{2,4}=-\beta_{2,4}=-1$, $\alpha_{3,4}=-\beta_{3,4}=-1$. Averaging all terms over periods in $\tau_{2}, \tau_{3}$, and $\tau_{4}$ (the first equation in the set which follows) and so on, and returning to the variable $t$, we readily subdivide Eq. (7) into four equations governing $\varphi_{1}\left(\tau_{1}, t\right), \varphi_{2}\left(\tau_{2}, t\right), \varphi_{3}\left(\tau_{3}, t\right)$, and $\varphi_{4}\left(\tau_{4}, t\right)$ :

$$
\begin{aligned}
& \frac{\partial^{2} \varphi_{i}}{\partial \tau_{i} \partial t}-c B \frac{\partial \varphi_{i}}{\partial \tau_{i}}=-\frac{\gamma+1}{2 c^{2}} \frac{\partial \varphi_{i}}{\partial \tau_{i}} \frac{\partial^{2} \varphi_{i}}{\partial \tau_{i}^{2}}, \\
&(i=1, \ldots 4) .
\end{aligned}
$$

Equations (8) are readily integrated:

$$
\frac{\partial \varphi_{i}}{\partial t}-c B \varphi_{i}=-\frac{\gamma+1}{4 c^{2}}\left(\frac{\partial \varphi_{i}}{\partial \tau_{i}}\right)^{2}, \quad(i=1, \ldots 4) .
$$

Equations (8), (9) are valid if $\varphi_{i}$ are periodic functions of $\tau_{i}$. In this case, the four wave constituents do not interact in the volume of the resonator in the leading order. Since $u_{x}=\sum_{i=1}^{4} u_{x, i}=\sum_{i=1}^{4} \frac{\partial \varphi_{i}}{\partial x}=$ $\frac{k_{x}}{\omega}\left(-\frac{\partial \varphi_{1}}{\partial \tau_{1}}-\frac{\partial \varphi_{2}}{\partial \tau_{2}}+\frac{\partial \varphi_{3}}{\partial \tau_{3}}+\frac{\partial \varphi_{4}}{\partial \tau_{4}}\right)$ and $u_{y}=\sum_{i=1}^{4} u_{y, i}=$ $\sum_{i=1}^{4} \frac{\partial \varphi_{i}}{\partial y}=\frac{k_{y}}{\omega}\left(\frac{\partial \varphi_{1}}{\partial \tau_{1}}-\frac{\partial \varphi_{2}}{\partial \tau_{2}}+\frac{\partial \varphi_{3}}{\partial \tau_{3}}-\frac{\partial \varphi_{4}}{\partial \tau_{4}}\right)$, the averaged over periods components of velocity are equal to zero for each of the four components of the field. The components of the individual velocities are described by the equations which follow from Eqs. (8):

$$
\begin{aligned}
\frac{\partial u_{x, i}}{\partial t}-c B u_{x, i} & =\chi_{i} \frac{\gamma+1}{2 c^{2}} \frac{\omega}{k_{x}} u_{x, i} \frac{\partial u_{x, i}}{\partial \tau_{i}} \\
\frac{\partial u_{y, i}}{\partial t}-c B u_{y, i} & =\delta_{i} \frac{\gamma+1}{2 c^{2}} \frac{\omega}{k_{y}} u_{y, i} \frac{\partial u_{y, i}}{\partial \tau_{i}}
\end{aligned}
$$

$\chi_{1}=\chi_{2}=1=-\chi_{3}=-\chi_{4}=1, \delta_{1}=\delta_{3}=-\delta_{2}=$ $-\delta_{4}=-1$. The equations which describe acoustic pressures belonging to each wave are

$$
\frac{\partial p_{i}}{\partial t}-c B p_{i}=\frac{\gamma+1}{2 c^{2} \rho_{0}} p_{i} \frac{\partial p_{i}}{\partial \tau_{i}}, \quad(i=1, \ldots 4),
$$

since the total acoustic pressure $p^{\prime} \approx-\rho_{0} \sum_{i=1}^{4}\left(\frac{\partial \varphi_{i}}{\partial \tau_{i}}\right)$. Equations (10), (11) relate to the waveforms without discontinuities and to the waveforms with discontinuities. The mean values of all components of velocity perturbations and acoustic pressure are zero in symmetric acoustic pulses. In the new dimensionless variables

$$
\begin{aligned}
\tau & =\omega t, & \eta_{i} & =\omega \tau_{i}, \\
i & =1, \ldots 4, & X & =\frac{\omega x}{c}, \\
Y & =\frac{\omega y}{c}, & K_{x} & =\frac{c k_{x}}{\omega}, \\
K_{y} & =\frac{c k_{y}}{\omega}, & b & =\frac{c B}{\omega}, \\
\mathbf{U} & =\frac{\mathbf{u} \exp (-b \tau)}{M c}, & P & =\frac{p^{\prime} \exp (-b \tau)}{M c^{2} \rho_{0}}, \\
\theta & =\exp (b \tau)-1, & &
\end{aligned}
$$

where $M$ is the Mach number, Eqs. (10), (11) take the forms $(i=1, \ldots 4)$

$$
\begin{aligned}
\frac{\partial U_{x, i}}{\partial \theta}-\frac{\chi_{i} G}{K_{x}} U_{x, i} \frac{\partial U_{x, i}}{\partial \eta_{i}} & =0 \\
\frac{\partial U_{y, i}}{\partial \theta}-\frac{\delta_{i} G}{K_{y}} U_{y, i} \frac{\partial U_{y, i}}{\partial \eta_{i}} & =0 \\
\frac{\partial P_{i}}{\partial \theta}-G P_{i} \frac{\partial P_{i}}{\partial \eta_{i}} & =0
\end{aligned}
$$


where $G=\frac{(\gamma+1) M}{2 b}$ is a non-dimensional quantity. The dimensionless wave numbers $K_{x}, K_{y}$ are real and positive, their ratio determines the angle between a beam and the boundaries of a resonator, and $K_{x}^{2}+K_{y}^{2}=1$. The solutions of (13) before formation of discontinuities are as follows (RUdenko, Soluyan, 2005):

$$
\begin{aligned}
U_{x} & =\sum_{i=1}^{4} U_{x, i}=2 \sum_{n=1}^{\infty} \frac{J_{n}\left(n \sigma_{x}\right) \sum_{i=1}^{4} \chi_{i} \sin \left(n \eta_{i}\right)}{n \sigma_{x}} \\
U_{y} & =\sum_{i=1}^{4} U_{y, i}=2 \sum_{n=1}^{\infty} \frac{J_{n}\left(n \sigma_{y}\right) \sum_{i=1}^{4} \delta_{i} \sin \left(n \eta_{i}\right)}{n \sigma_{y}} \\
P & =\sum_{i=1}^{4} P_{i}=2 \sum_{n=1}^{\infty} \frac{J_{n}(n \sigma) \sum_{i=1}^{4} \sin \left(n \eta_{i}\right)}{n \sigma}
\end{aligned}
$$

where

$$
\sigma_{x}=\frac{G \theta}{K_{x}}, \quad \sigma_{y}=\frac{G \theta}{K_{y}}, \quad \sigma=G \theta .
$$

The boundary conditions for an impenetrable flow at the boundaries of a resonator $x=0, x=L_{x}, y=0$ and $y=L_{y}$ are given by equalities

$$
\begin{aligned}
& U_{x}(X=0, Y, t)=U_{y}\left(X=\frac{2 n_{x} \pi}{K_{x}}, Y, t\right)=0, \\
& U_{y}(X, Y=0, t)=U_{y}\left(X, Y=\frac{2 n_{y} \pi}{K_{y}}, t\right)=0 .
\end{aligned}
$$

The length and width of a rectangular resonator equal integer number of longitudinal or transversal wavelengths, $L_{x}=n_{x} \lambda_{x}, L_{y}=n_{y} \lambda_{y}$, that in fact determines the spectrum of longitudinal and vertical components of wave numbers. An acoustic pressure and vertical velocity in a rectangular resonator before formation of a discontinuity in an acoustically active gas with $B>0$ are shown in Figs. 1 and 2. In evaluations in accordance with (14), only ten first terms were taken into account. Numerical evaluations undertaken by the author reveal practically indistinguishable results starting from the first five terms in the series. The series is quickly convergent. The dimensionless time of shock formation $T_{\text {saw }}$ for the profile of the vertical velocity is $\frac{1}{b} \ln \left(1+\frac{K_{y}}{G}\right)$, the one for the horizontal velocity is $\frac{1}{b} \ln \left(1+\frac{K_{x}}{G}\right)$, and the one for acoustic pressure equals $\frac{1}{b} \ln \left(1+\frac{1}{G}\right)$.

For dimensionless times $\sigma_{y}$ larger than $\pi / 2$, the dimensionless velocities $U_{y, i}(i=1, \ldots 4)$ take the form of the saw-tooth shapes consisting of straight-line parts,

$$
U_{y, i}=-\frac{\delta_{i} \tau_{i}}{1+G \theta / K_{y}} \quad \text { if } \quad-\pi \leq \tau_{i}<\pi .
$$

Individual dimensionless acoustic pressures $P_{i}(i=$ $1, \ldots 4)$ also take the saw-tooth form, if $\sigma>\pi / 2$ :

$$
P_{i}=-\frac{\tau_{i}}{1+G \theta} \quad \text { if } \quad-\pi \leq \tau_{i}<\pi .
$$

The peak magnitude $p_{i}^{\prime}$ does not tend to zero when time increases in an acoustically active gas. The nonlinear attenuation at the front of a saw-tooth wave is suppressed by enlargement of the sound intensity. The limit value of dimensionless peak magnitude of every part of the vertical velocity $u_{y, i}$ is $\frac{2 \pi R_{y} b c}{\gamma+1}$, and that of the acoustic pressure is $\frac{2 \pi b c \rho_{0}^{2}}{\gamma+1}$, when $\theta \rightarrow \infty$. In the equilibrium regime, normal dispersion and attenuation support nonlinear attenuation, and peak values quickly tend to zero. In this case, discontinuity in the profile of the vertical velocity does not form for a large enough attenuation, that is, for $\frac{K_{y}}{G} \leq-1$.

The peak dimensionless magnitude of the vertical velocity at the same times as in the Fig. 3, for $b=-0.01$ and $K_{y}=0.2$, equals 0.2 at $\tau=2 T_{\text {saw }}$ and 0.002 at $\tau=20 T_{\text {saw }}$, respectively. $T_{\text {saw }}$ is evaluated at $b=0.01$. For $b=-0.01$ and $K_{y}=0.8$, discontinuity does not form at all. Figure 4 shows the dis-

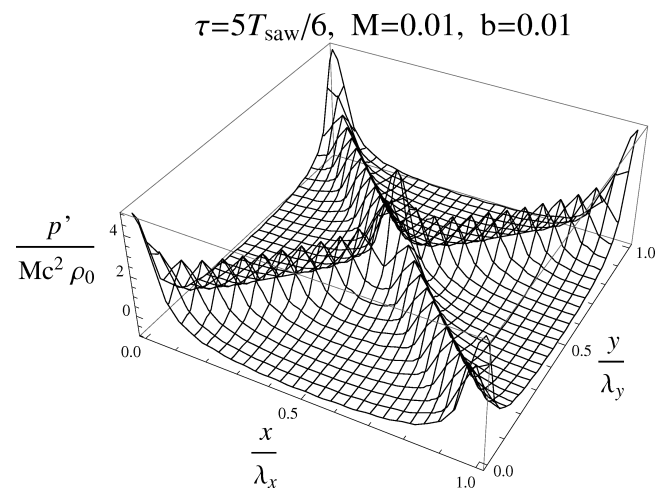

Fig. 1. Dimensionless acoustic pressure $p^{\prime} /\left(M c^{2} \rho_{0}\right)$ in a resonator at different dimensionless times before formation of discontinuity. For $M=0.01, b=0.01, \gamma=1.4$, the dimensionless time of discontinuity formation $T_{\text {saw }}$ equals 61 . 

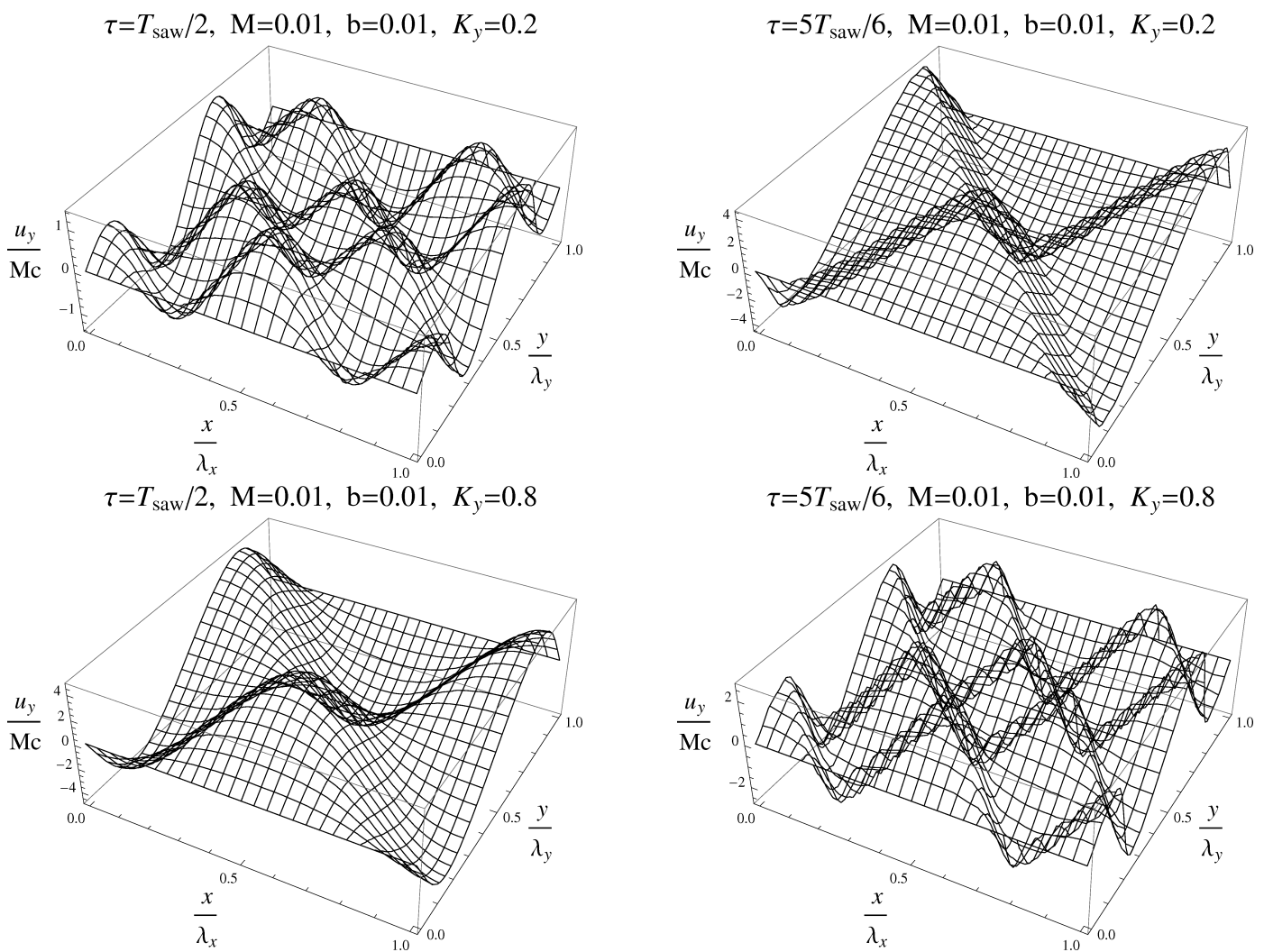

Fig. 2. Dimensionless vertical velocity $u_{y} /(M c)$ in a resonator at different dimensionless times and for different dimensionless values of $K_{y}$ before formation of discontinuity. For $M=0.01, b=0.01, \gamma=1.4$, the dimensionless time of discontinuity formation $T_{\text {saw }}$ equals 15 if $K_{y}=0.2$ and 51 if $K_{y}=0.8$.
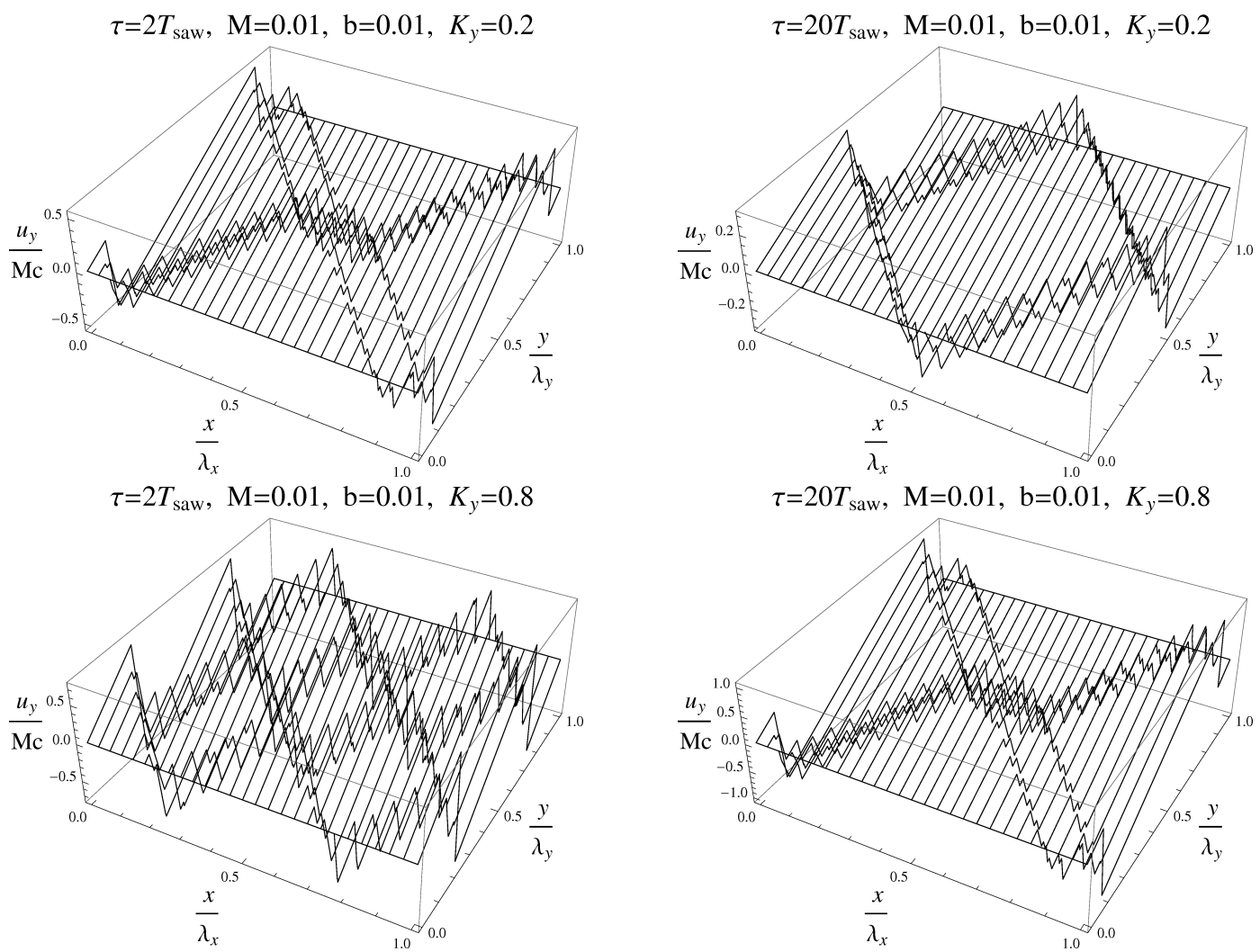

Fig. 3. Dimensionless vertical velocity $u_{y} /(M c)$ in a resonator at different dimensionless times and for different dimensionless $K_{y}$ in a saw-tooth wave. For $M=0.01, b=0.01, \gamma=1.4$, the dimensionless time of saw-tooth shape formation $T_{\text {saw }}$ equals 23 if $K_{y}=0.2$ and 72 if $K_{y}=0.8$. 

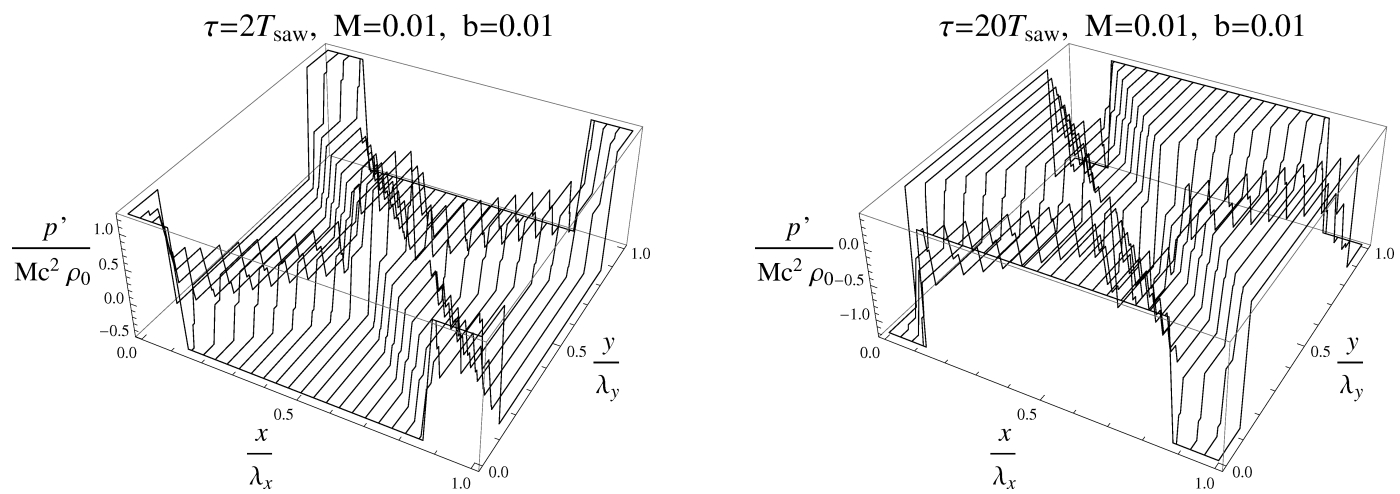

Fig. 4. Dimensionless acoustic pressure $p^{\prime} /\left(M c^{2} \rho_{0}\right)$ in a resonator at different dimensionless times in a saw-tooth wave. For $M=0.01, b=0.01, \gamma=1.4$, the dimensionless time of the saw-tooth shape formation $T_{\text {saw }}$ equals 84 .

tribution of dimensionless acoustic pressure in a volume of a resonator at different dimensionless times. The dimensionless time of discontinuity formation in a pressure standing wave, $T_{\text {saw }}$, equals 84 . The magnitude of dimensionless acoustic pressure achieves 0.1 for $b=-0.01$ and $\tau=2 T_{\text {saw }}$ and $2 \cdot 10^{-8}$ for $b=-0.01$ and $\tau=20 T_{\text {saw }}$, respectively.

\section{Acoustic heating}

An excess temperature $T_{\text {ent }}$ which specifies the non-wave entropy mode in the field of acoustic planar waves which do not interact, is governed by the leading-order equation (PERELOMOVA, 2012)

$$
\frac{1}{T_{0}} \frac{\partial T_{\mathrm{ent}}}{\partial \tau}=-b M^{2} \exp (2 b \tau)(\gamma-1) \sum_{i=1}^{4} \overline{P_{i}^{2}}
$$

in the case of vibrationally excited gases, and

$\frac{1}{T_{0}} \frac{\partial T_{\text {ent }}}{\partial \tau}=-b M^{2} \exp (2 b \tau) \frac{(\gamma-1)(\gamma+2)}{\gamma} \sum_{i=1}^{4} \overline{P_{i}^{2}}$

in the case of chemically reacting gases (PERELOMOVA, PelC-Garska, 2014; 2011), where the top line denotes the average over the sound period, and $T_{0}$ is an unperturbed temperature of a gas. Before the formation of discontinuity, the rate of heat release varies as $b \exp (-2 b \tau)$, since the averaged squared dimensionless pressure $\overline{P_{i}^{2}}$ equals $\overline{P_{i}^{2}}=-\overline{\frac{\partial P_{i}}{\partial \tau_{i}} \int P_{i} \mathrm{~d} \tau_{i}}=0.5$. It depends on the sign of $b$ : the temperature of the medium increases if $b<0$ and decreases otherwise. In the case of the saw-tooth wave, $\overline{P_{i}^{2}}=\frac{\pi^{2}}{3(1+G(\exp (b \tau)-1))^{2}}$, and the acoustic heating is described by equation

$\frac{1}{T_{0}} \frac{\partial T_{\text {ent }}}{\partial \tau}=-4 M^{2} \pi^{2}(\gamma-1) \frac{b \exp (2 b \tau)}{3\left(1+G(\exp (b \tau)-1)^{2}\right)}$ in the chemically reacting gases, and by equation

$$
\begin{aligned}
\frac{1}{T_{0}} \frac{\partial T_{\text {ent }}}{\partial \tau}= & -4 M^{2} \pi^{2} \frac{(\gamma-1)(\gamma+2)}{3 \gamma} \\
& \cdot \frac{b \exp (2 b \tau)}{\left(1+G(\exp (b \tau)-1)^{2}\right)}
\end{aligned}
$$

in the gases with excited internal degrees of a molecule freedom. In the non-equilibrium regime, enlargement in the sound magnitude is followed by cooling of a medium. The sound wave "takes energy" from the background making it cooler.

\section{Concluding remarks}

The peculiarities of a periodic acoustic field in a rectangular resonator filled with some relaxing medium, which may be acoustically active, are studied. If the mean value of acoustic pressure in the incident and reflected waves is zero, they do not interact in the leading order in a volume of the resonator. That allows to consider the incident and reflected waves individually. In the equilibrium regime, discontinuity may not form at all for large enough attenuations connected with relaxation in a gas. If the saw-tooth wave forms, the peak acoustic pressure rapidly decreases towards zero. Vice versa, an anomalous increase in the sound amplitude, along with the nonlinear attenuation, result in the settling of the peak magnitude in the shock wave in a non-equilibrium gas. The conclusions concern propagation of the planar wave in a free space, in waveguides (PERELOMOVA, 2012) and resonators. In the two last cases, the discrete set of wave numbers is determined by the boundary conditions. There appear the additional travelling nodal points in the velocity profile of standing waves in a flat resonator (one travelling point between two static ones). There are no additional nodal points in the profile of the acoustic pressure, but their coordinates vary with time periodically. That has been discovered with respect to gases with irreversible chemical reactions 
in (Perelomova, Pelc-Garska, 2014). The conclusions are valid also in a rectangular resonator. In contrast, the nodal points in the standing waves in Newtonian fluids are static.

The slowly developing in time modes which are induced in the sound field in the non-equilibrium gases, also behave atypically. The anomalous cooling of a medium and streaming (with streamlines inverted as compared with direction of streamlines in a Newtonian fluid) has been recently considered in reference to aperiodic and periodic in time sound beams, including beams with discontinuities in unbounded volumes of acoustically active gases (Molevich, 2001; PERELOmova, Pelc-Garska, 2011; Perelomova, 2012). An increase (or decrease) in the temperature of a gas does not disturb the boundary conditions in resonators (16), since the velocity associated with the entropy mode, is zero in the leading order. The squared sound velocity $c^{2}$ is proportional to the mean temperature, which makes the set of longitudinal and transversal wave numbers vary in the course of time in order to hold the sound frequency constant. Hence, they enlarge in the acoustically active gas (and get smaller otherwise) proportionally to the square root of the initial sound intensity and $B$. The effects connected with the first and second viscosities and thermal conduction, are not considered in this study. They may be readily included. Newtonian attenuation alone makes the peak pressure in the saw-tooth waves in the non-equilibrium regime to decrease; at large times, it tends to zero. Account for Newtonian attenuation may prevent formation of discontinuity in the acoustically active gas.

\section{References}

1. Bauer H.J., Bass H.E. (1973), Sound amplification from controlled excitation reactions, Phys. Fluids, 16, 988-997, http://dx.doi.org/10.1063/1.1694494.

2. Biwa T., Yazaki T. (2010), Observation of energy cascade creating periodic shock waves in a resonator, J. Acoust. Soc. Am., 127, 3, 1189-1192, http://dx.doi.org/10.1121/1.3291029.

3. Chester W. (1964), Resonant oscillations in closed tubes, J. Fluid Mech., 18, 44-64, http://dx.doi.org/10.1017/S0022112064000040.

4. Chu B.T. (1970), Weak nonlinear waves in nonequilibrium flows, [in:] Nonequilibrium flows, Wegener P.P. [Ed.], vol. 1, part 2, Marcel Dekker, New York.

5. Clarke J.F., McChesney A. (1976), Dynamics of relaxing gases, Butterworth, UK.

6. Hamilton M., Morfey C. (1998), Model equations, [in:] Nonlinear Acoustics, Hamilton M., Blackstock D. [Eds.], pp. 41-63, Academic Press, New York.
7. Kaner V.A., Rudenko O.V., Khokholov R.V. (1977) Theory of nonlinear oscillations in acoustic resonators, Sov. Phys. Acoust., 23, 5, 432-437.

8. Keller J.J. (1977), Nonlinear acoustic resonances in shock tubes with varying cross-sectional area, J. Appl. Math. Phys., 28, 107-122, http://dx.doi.org/10.1007/BF01590712.

9. Makaryan V.G., Molevich N.E. (2007), Stationary shock waves in nonequilibrium media, Plasma Sources Sci. Technol., 16, 124-131.

10. Molevich N.E. (2001), Sound amplification in inhomogeneous flows of nonequilibrium gas, Acoustical Physics, 47, 1, 102-105, http://dx.doi.org/10.1134/1.1340086.

11. Mortell M.P., Mulchrone K.F., Seymour B.R. (2009), The evolution of macrosonic standing waves in a resonator, International Journal of Engineering Sience, 47, 11-12, 1305-1314, http://dx.doi.org/10.1016/j.ijengsci.2008.10.012.

12. Ochmann M. (1985), Nonlinear resonant oscillations in closed tubes - an application of the averaging method, J. Acoust. Soc. Am., 77, 1, 61-66, http://dx.doi.org/10.1121/1.391901.

13. Osipov A.I., Uvarov A.V. (1992), Kinetic and gasdynamic processes in nonequilibrium molecular physics, Sov. Phys. Usp., 35, 11, 903-923, http://dx.doi.org/10.1070/PU1992v035n11ABEH002275.

14. PARKer D.F. (1972), Propagation of damped pulses through a relaxing gas, Phys. Fluids, 15, 256-262, http://dx.doi.org/10.1063/1.1693902.

15. Rudenko O.V., Soluyan S.I. (2005), Theoretical foundations of nonlinear acoustics, Consultants Bureau, New York, DOI: 10.1002/jcu.1870060222.

16. Perelomova A. (2010), Nonlinear generation of nonacoustic modes by low-frequency sound in a vibrationally relaxing gas, Canadian Journal of Physics, 88, 4, 293-300, doi:10.1139/P10-011.

17. Perelomova A. (2012), Standing acoustic waves and relative nonlinear phenomena in a vibrationally relaxing gas-filled resonator, Acta Acustica, 98, 713-721, http://dx.doi.org/10.3813/AAA.918552.

18. Perelomova A., Pelc-Garska W. (2014), Standing waves and acoustic heating (or cooling) in resonators filled with chemically reacting gas, Archives of Acoustics, 39, 3, 403-410, doi: 10.2478/aoa-2014-0044.

19. Perelomova A., Pelc-Garska W. (2011), Non-wave variations in temperature caused by sound in a chemically reacting gas, Acta Physica Polonica A, 120, 3, $455-461$.

20. Zeldovich Ya.B., Raizer Yu.P. (1966), Physics of shock waves and high temperature hydrodynamic phenomena, Academic Press, New York. 